\title{
A Learning-to-Rank-Based Investment Portfolio Optimization Framework for Smart Grid Planning
}

\author{
Wenxin Zhao ${ }^{1}$, Xubin $\mathrm{Liu}^{2 *}$, Yujie $\mathrm{Wu}^{1}$, Tao Zhang ${ }^{1}$ and Luao Zhang ${ }^{1}$ \\ ${ }^{1}$ College of Electrical and Information Engineering, Hunan University, Changsha, China, ${ }^{2}$ School of Automation, Central South \\ University, Changsha, China
}

Keywords: machine learning, power grid planning, investment portfolio, infrastructure project, combinatorial optimization

\section{INTRODUCTION}

Power grid planning is to formulate a strong, efficient, economical, and sustainable power grid by scientifically implementing the investment portfolio optimization of infrastructure projects to ensure the stable and safe operation of smart grid and realize the rational and optimal allocation of energy resources (Liu et al., 2017; Li et al., 2018). Power grid infrastructure projects exhibit the characteristics of large investment, long construction period, and a huge number of projects, and the construction status is closely related to the national economy and people's livelihood (Wu

Edited by: Jian Zhao,

Shanghai University of Electric Power,

China

Reviewed by:

Bo Yang,

Kunming University of Science and

Technology, China

$X i L u$,

Southeast University, China

*Correspondence:

Xubin Liu

liuxubin@csu.edu.cn

Specialty section:

This article was submitted to

Process and Energy Systems

Engineering,

a section of the journal

Frontiers in Energy Research

Received: 11 January 2022

Accepted: 24 January 2022

Published: 14 February 2022

Citation:

Zhao W, Liu X, Wu Y, Zhang T and

Zhang $L$ (2022) A Learning-to-Rank-

Based Investment Portfolio

Optimization Framework for Smart

Grid Planning.

Front. Energy Res. 10:852520.

doi: 10.3389/fenrg.2022.852520 et al., 2019). Therefore, it is crucial to choose a reasonable and optimum investment portfolio for smart grid planning.

The investment portfolio optimization of power grid infrastructure projects is a non-convex, multi-period coupling, strong constraint, and multi-objective portfolio optimization problem. When making a decision, it is necessary to comprehensively consider the relevant decision index standards, related constraints, and the expected target requirements (Wu et al., 2019). It is a complex systemic decision-making work that requires the use of system science, decision science, optimization modeling, and other related content to be better completed (Zhao et al., 2021). The core is to establish a scientific and objective investment portfolio optimization model rather than purely administrative decision-making (Liu et al., 2017; Zhang et al., 2021). Consequently, it is vital to comprehensively consider factors such as synthetical benefits, construction time sequence, policy, and market risks to develop an intelligent and refined planning framework for the investment portfolio optimization of power grid infrastructure projects and form a more scientific and effective means of auxiliary decision-making for power grid development.

\section{INVESTMENT PORTFOLIO OPTIMIZATION FOR POWER GRID PLANNING}

The investment portfolio optimization of power grid infrastructure projects requires research and analysis of the company's strategic goals of power grid development, operation, maintenance, marketing, dispatch, and other multi-service data and forms, as well as the data characteristics of the internal and external policies and economic, social, environmental, and other multiple regulatory information. Furthermore, it needs to coordinate the multiple dimensions of high-quality development of power grid efficiency, eco-friendly growth, service quality, and operating performance (Liu et al., 2017; Li et al., 2018; Wu et al., 2019). Moreover, it is necessary to fully consider the factors of the current situation and future progress of the power grid in different 


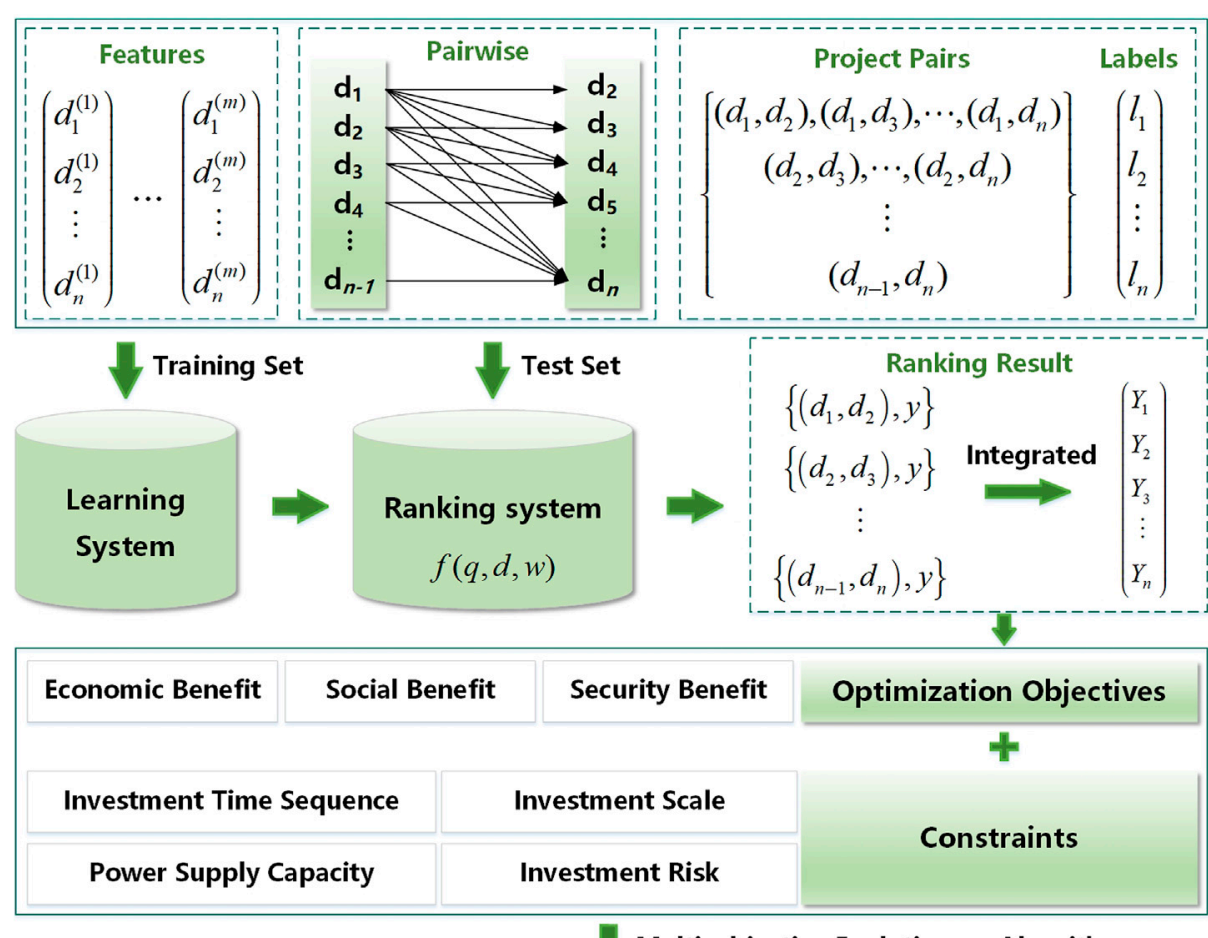

Multi-objective Evolutionary Algorithm

Investment Portfolio of Power Grid Infrastructure Project

FIGURE 1 | Proposed L2R-based investment portfolio optimization framework.

provinces. It is also complicated and demanding work with a large amount of data, workload, and high technical requirements (Liu et al., 2020).

In power grid investment decision-making, decisionmakers often have to face numerous practical data, choices, and risks, and it is difficult to determine the mutual influence of various factors. In addition, the power grid investment decision-making problem generally consists of a series of decision-making options. Each of the decision-making options is related to the other, which requires decisionmakers to analyze the overall situation (Zhao et al., 2021). Most of the existing investment portfolio optimization strategies are from the perspective of problem-oriented research, based on the known investment demands and investment capabilities of the enterprise, referring to the previous investment allocation, to allocate the investment scale and investment structure of each unit (Peng and Zhang, 2015; Li et al., 2021). The objective function and constraints are not considered enough comprehensive and systematic, and model boundary conditions and project priority ranking rely mainly on expert experience to make subjective decisions, lacking the recognition and consideration of objective factors (Guelpa et al., 2019; Liu et al., 2020; Ma et al., 2020).

The features of power grid projects are reflected in many aspects such as technology, economy, society, and practicality.
The criticality of the features needs to comprehensively consider multiple dimensions and factors such as engineering properties and project necessity, which increases the complexity of defining and extracting key features (Yang et al., 2016; Zhao et al., 2021). Besides, the emerging changes brought about by the advanced development situation will not affect a certain aspect in isolation and will generally form a linkage effect of multiple factors (Liu et al., 2017), which further increases the difficulty of analyzing and extracting new key features, making it more difficult to propose a feature index system. To face the recent emerging problems arising from power grid planning, it is urgent to use machine learning to formulate an investment performance evaluation model covering traditional investment efficiency improvement (Ma et al., 2020). Realizing the intelligent upgrade of traditional investment decision-making technology and making full use of the precise positioning of power grid investment can ensure the healthy and sustainable development of the power grid and contribute a powerful boost to economic development.

\section{LEARNING-TO-RANK METHODOLOGY}

Machine learning as an emerging technology has been widely used in all aspects of life to obtain effective information. There are 
many important overlaps between the fields of information retrieval and machine learning. Learning-to-rank, as a product of the combination of machine learning and information retrieval technology, is an important application branch of machine learning ( $\mathrm{Li}, 2014)$. In the field of information retrieval, the search results are ranked and recommended to obtain the most desired results for users. The main way to establish a traditional search ranking model is to rely on manual and continuous experiments to determine the parameters of functions with relevance scoring (Li, 2014; Xu et al., 2019). The learning-to-rank (L2R) algorithms based on machine learning have made great improvements. The final ranking formula in the L2R algorithms is obtained by automatic learning, while people only need to provide relevant training data for the L2R algorithm.

$\mathrm{L} 2 \mathrm{R}$ is a process of supervised learning, including training and testing. The machine learning ranking system consists of four steps: dataset acquisition, ranking model training, test set testing, and model application. A typical L2R model consists of two parts: learning system and ranking system. The L2R algorithms obtain the optimal ranking model from the training data through the learning system. In other words, the L2R model minimizes the value of the loss function ( $\mathrm{Li}, 2014$; $\mathrm{Xu}$ et al., 2019). Then, through the ranking system, the test set is predicted and ranked with the trained optimal ranking model, and the loss function value of the test sample is required to be lower than the set minimum target value ( $\mathrm{Li}, 2014$; Xu et al., 2019). In addition, making a correct judgment on the performance of the L2R system is a very important issue. Generally, there is a specified evaluation index to judge the pros and cons of the model. Currently, the main ranking evaluation indexes include mean average precision (MAP), recall $(\mathrm{R})$, mean reciprocal rank (MRR), expected reciprocal rank (ERR), and normalized discounted cumulative gain (NDCG) (Li, 2014; Xu et al., 2019; Xia et al., 2021).

Based on the difference in the input training samples, L2R algorithms can be divided into three categories: pointwise, pairwise, and listwise. The algorithms of pointwise and pairwise take a single document and a pair of documents as the input of the training samples, respectively, converting the rank of retrieval results into regression or classification problems (Li, 2014). However, the listwise algorithms differ from the former two, taking the retrieval results obtained by the user's query as a whole and serving as a sample for training. Among them, the pairwise $\mathrm{L} 2 \mathrm{R}$ algorithms turn the ranking problem into the judgment on the order for the sample pairs. For any two samples with different labels in the sample set, they can be combined into a sample pair $\left(x_{i}, x_{j}\right)$, which is labeled based on the relative relationship between the two samples. A sample pair has only two relative order relationships; that is, sample $x_{i}$ is ranked before or after sample $x_{j}$. Therefore, the ranking problem can naturally be transformed into the judgment on the relationship between any two samples, and the judgment on the order of sample pairs becomes a very typical binary classification problem. The commonly used L2R algorithms of pairwise include RankBoost, RankNet, LambdaRank, and LambdaMART (Li, 2014; Xia et al., 2021).

\section{LEARNING-TO-RANK-BASED INVESTMENT DECISION-MAKING FRAMEWORK}

Power grid investment decision-making is a complex process that needs to consider the objectives of the economic, social, and security benefits and meet the constraints of capital investment, project construction time sequence, and power demand (Peng and Zhang, 2015; Liu et al., 2020). It is difficult to reproduce this process with a simple end-to-end machine learning method. On the one hand, power grid investment benefit evaluation indexes mainly include economic, social, and safety evaluation (Yang et al., 2016; Guelpa et al., 2019). With the increase in evaluation indexes, the objective space increases exponentially, and there are complex connections among the objectives, resulting in high requirements for the multi-objective optimization ability of machine learning algorithms. On the other hand, the investment portfolio optimization of power grid infrastructure projects has a huge number of samples and abundant features. The traditional manual selection method cannot excavate the correlation of the features in the case of complex information on infrastructure projects.

In order to cope with these challenges, a learning-to-rankbased investment portfolio optimization framework of power grid infrastructure projects is proposed, and it's presented in Figure 1. L2R with autonomous learning can clarify the correlation among complex features from different infrastructure projects. The key steps of this framework are as follows:

1) The collected data are preprocessed to form standardized feature parameters, including project properties, construction date, and construction scale, and then training and test sets are created.

2) According to the pairwise compound mode of project pairs, all samples needed for binary classifier training are formed.

3) A ranking model based on the pairwise $L 2 R$ is formulated to transform the ranking of projects into a binary classification problem, which mines the ranking relations among partial projects.

4) When making predictions, the final ranking function obtained by model training will give the calculated scores of the project pairs.

5) Partial ordering relations of all project pairs are integrated to learn the ranking laws of the overall candidate itemset for selection.

The investment portfolio optimization objective of the power grid infrastructure project is to minimize the comprehensive benefits (Peng and Zhang, 2015; Liu et al., 2020; Ma et al., 2020). The traditional optimization decisionmaking method is usually based on the investment performance evaluation index system to score the projects. In contrast, the L2R-based investment portfolio optimization ranking model will replace this index system, optimizing the investment portfolio according to the ranking results under different benefit orientations. Furthermore, a multi-objective 
model of investment portfolio optimization is formed considering the constraints of investment scale, investment time sequence, power supply capacity, and investment risk. In order to solve this model, multi-objective evolutionary algorithms are commonly used to obtain a scientific investment portfolio of power grid infrastructure projects (Huang et al., 2020; Zhang and Li, 2020), providing a basis for the compilation of the grid's annual investment planning.

\section{DISCUSSION AND CONCLUSION}

L2R aims to mine the potential correlated features in samples and excavate the underlying objective laws from the historical optimal portfolio, which fits well with the ranking and investment portfolio optimization scenario of power grid infrastructure projects. Therefore, research on the L2R-based investment portfolio optimization method of power grid infrastructure projects is necessary, which is of great significance for learning the in-depth regular patterns, timely discovering the existing weak links, and making up for the shortcomings in the development of power grids. The intellectualized closed-loop

\section{REFERENCES}

Guelpa, E., Bischi, A., Verda, V., Chertkov, M., and Lund, H. (2019). Towards Future Infrastructures for Sustainable Multi-Energy Systems: A Review. Energy 184, 2-21. doi:10.1016/j.energy.2019.05.057

Huang, Z., Fang, B., and Deng, J. (2020). Multi-objective Optimization Strategy for Distribution Network Considering V2G-Enabled Electric Vehicles in Building Integrated Energy System. Prot. Control. Mod. Power Syst. 5 (1), 48-55. doi:10. 1186/s41601-020-0154-0

Li, H. (2014). Learning to Rank for Information Retrieval and Natural Language Processing. Second Edition. Williston, Vermont, United States: Morgan \& Claypool.

Li, J., Chen, L., Xiang, Y., Li, J., and Peng, D. (2018). Influencing Factors and Development Trend Analysis of China Electric Grid Investment Demand Based on a Panel Co-integration Model. Sustainability 10 (1), 256. doi:10.3390/ su10010256

Li, J., Chen, W., Chen, Y., Sheng, K., Du, S., Zhang, Y., et al. (2021). A Survey on Investment Demand Assessment Models for Power Grid Infrastructure. IEEE Access 9, 9048-9054. doi:10.1109/ACCESS.2021.3049601

Liu, J., Gao, J., and Wang, Y. (2017). "Research on the Principles and Strategies of Power Grid Investment under the New Situation of Power Industry Reform," in Proceedings of the 2017 Chinese Automation Congress (CAC), Jinan, China, October 2017, 6274-6278. doi:10.1109/CAC.2017.8243908

Liu, Y., Ma, Q., Xu, C., Liu, H., and Wang, Z. (2020). "Investment Optimization Model for Distribution Networks Projects with Risk and Efficiency Constraints," in Proceedings of the 2020 IEEE 4th Conference on Energy Internet and Energy System Integration (EI2), Wuhan, China, 30 Oct.-1 Nov. 2020, 2047-2052. doi:10.1109/EI250167.2020.9346991

Ma, Y., Han, R., and Wang, W. (2020). Prediction-Based Portfolio Optimization Models Using Deep Neural Networks. IEEE Access 8, 115393-115405. doi:10. 1109/ACCESS.2020.3003819

Peng, W., and Zhang, J. (2015). "Investment Portfolio Optimization of Power Grid Projects Based on Niche Genetic Algorithms," in Proceedings of the 2015 11th International Conference on Natural Computation (ICNC), Zhangjiajie, China, August 2015, 1170-1175. doi:10.1109/ICNC.2015.7378157

Wu, W., Li, M., Yan, T., Huang, P., Lu, X., and Wang, Z. (2019). “Distribution Network Project Portfolio Optimization Decision Model Based on Power Demand Matching," in Proceedings of the 2019 IEEE PES Asia-Pacific Power and Energy Engineering Conference (APPEEC), Macao, China, December 2019, 1-5. doi:10.1109/APPEEC45492.2019.8994475 investment decision-making strategy of power grid infrastructure project can assist the corporate headquarters and various provinces, prefectures, cities, and districts to implement intelligent management of power grid investment and achieve reliable, convenient, and efficient investment portfolio optimization of large-scale and massive power grid infrastructure projects, improving the scientificity, efficiency, and accuracy of the investment management of power grids at all levels.

\section{AUTHOR CONTRIBUTIONS}

Writing the original draft and editing: WZ. Conceptualization: XL. Formal analysis: YW. Visualization and contribution to the discussion of the topic: TZ and LZ.

\section{FUNDING}

This work was supported by the Hunan Natural Science Foundation of China under Grant 2021JJ10019.

Xia, W., Ren, Z., Li, H., and Hu, B. (2021). A Power Fluctuation Evaluation Method of PV Plants Based on RankBoost Ranking. Prot. Control. Mod. Power Syst. 6 (3), 347-356. doi:10.1186/s41601-021-00205-y

Xu, B., Lin, H., Lin, Y., Diao, Y., Yang, L., and Xu, K. (2019). Extracting Emotion Causes Using Learning to Rank Methods from an Information Retrieval Perspective. IEEE Access 7, 15573-15583. doi:10.1109/ACCESS. 2019.2894701

Yang, F., Zhang, D., and Sun, C. (2016). China's Regional Balanced Development Based on the Investment in Power Grid Infrastructure. Renew. Sustain. Energ. Rev. 53, 1549-1557. doi:10.1016/j.rser.2015.09.066

Zhang, K., Zhou, B., Or, S. W., Li, C., Chung, C. Y., and Voropai, N. I. (2021). Optimal Coordinated Control of Multi-Renewable-To-Hydrogen Production System for Hydrogen Fueling Stations. IEEE Trans. Ind. Applicat., 1. doi:10. 1109/TIA.2021.3093841

Zhang, M., and Li, Y. (2020). Multi-Objective Optimal Reactive Power Dispatch of Power Systems by Combining Classification-Based Multi-Objective Evolutionary Algorithm and Integrated Decision Making. IEEE Access 8, 38198-38209. doi:10.1109/ACCESS.2020.2974961

Zhao, K., Dai, Y., Ji, Y., and Jia, Z. (2021). Decision-Making Model to Portfolio Selection Using Analytic Hierarchy Process (AHP) with Expert Knowledge. IEEE Access 9, 76875-76893. doi:10.1109/ACCESS.2021. 3082529

Conflict of Interest: The authors declare that the research was conducted in the absence of any commercial or financial relationships that could be construed as a potential conflict of interest.

Publisher's Note: All claims expressed in this article are solely those of the authors and do not necessarily represent those of their affiliated organizations or those of the publisher, the editors, and the reviewers. Any product that may be evaluated in this article, or claim that may be made by its manufacturer, is not guaranteed or endorsed by the publisher.

Copyright (c) 2022 Zhao, Liu, Wu, Zhang and Zhang. This is an open-access article distributed under the terms of the Creative Commons Attribution License (CC BY). The use, distribution or reproduction in other forums is permitted, provided the original author(s) and the copyright owner(s) are credited and that the original publication in this journal is cited, in accordance with accepted academic practice. No use, distribution or reproduction is permitted which does not comply with these terms. 